\title{
TAGUNG
}

\section{Der 80\%-Mythos auf dem Prüfstand: Wie europäisch ist die nationale Politik?}

\author{
Katharina Joho*
}

Im Jahr 1988 äußerte der damalige Präsident der Europäischen Kommission Jacques Delors die berühmte und vielzitierte These, dass in zehn Jahren 80 Prozent der nationalen Wirtschaftsgesetzgebung, vielleicht auch der steuerlichen und sozialen, gemeinschaftlichen Ursprungs sein würden. ${ }^{1}$ Diese These hat in den folgenden Jahren die politische Debatte über das Thema der europäischen Integration und ihren Einfluss auf die Systeme der Mitgliedstaaten geprägt. Sie wurde von vielen Politikern als Argument dafür verwendet, vor dem identitätsgefährdenden Einfluss Brüssels auf die Mitgliedstaaten zu warnen. Doch wie realistisch ist der sogenannte $80 \%$-Mythos und inwiefern ist er überhaupt zu überprüfen? Ist das Phänomen der Europäisierung tatsächlich in Prozenten messbar? Diese Fragen beschäftigen die Forschung vor allem in den Sozial-, Rechts- und Wirtschaftswissenschaften nun schon seit einiger Zeit und sie sind - wie so vieles - deutlich komplexer, als die Aussage von Delors vermuten ließe. Trotz der ansteigenden Zahl an Forschungsbeiträgen zu diesem Thema scheint das Phänomen der Europäisierung nicht klarer, sondern unklarer geworden zu sein. Claudio Radaelli äußerte im Rahmen der englischsprachigen Tagung mit dem Titel „Europäisierung messen jenseits des 80\%-Mythos“ die Frage: „Wissen wir immer weniger über das Phänomen der Europäisierung, je mehr wir darüber forschen?"

\section{Measuring Europeanization of Public Policies Beyond the 80\%-Myth}

Tagung des Wissenschaftszentrums Berlin für Sozialforschung (WZB) in Zusammenarbeit mit dem Arbeitskreis Europäische Integration e.V. (AEI)

mit Unterstützung der Europäischen Kommission

Berlin, 27./28. Februar 2009

Thematic Context: Why Measuring Europeanization?

Causal Approaches in the Context of Europeanization Research: A Meta-Analytic Perspective

Prof. Dr. Claudio Radaelli, University of Exeter

Measuring the Europeanization of LawMaking in Institutional Context: From Executive Dominance to Parliamentary Scrutiny

Prof. Dr. Thomas König, MZES, Mannheim Centre for European Social Research

Measuring Europeanization to Understand the Transformation of the State? Identifying European Causes of Market-Oriented Modernisation

Prof. Dr. Hans-Jürgen Bieling, University of Hamburg

Comment/Discussion

Prof. Dr. Gunnar Folke Schuppert, WZB, Social Science Research Center Berlin

* Dipl.-Pol. Katharina Joho, Wissenschaftliche Mitarbeiterin an der Arbeitsstelle Europäische Integration und Koordinatorin der Internationalen Dienste am Otto-Suhr-Institut für Politikwissenschaft der Freien Universität Berlin.

1 Vgl. Rede von Jacques Delors am 06.07.1988 vor dem Europäischen Parlament, in: Amtsblatt der EG. Verhandlungen des Europäischen Parlaments, Nr. 2-367 vom 6. Juli 1988, S. 154-157, hier S. 157. 
Wie vielschichtig und vielfältig die Europäisierungsforschung ist, wurde denn auch auf der gemeinsam vom Wissenschaftszentrum Berlin und dem Arbeitskreis Europäische Integration organisierten zweitägigen Tagung deutlich. Auf vier verschiedenen Panels diskutierten Wissenschaftler der Politik-, Rechts- und Wirtschaftswissenschaften unter besonderer Berücksichtigung quantitativer Ansätze die Grenzen und aktuellen Probleme der Europäisierungsforschung.

Das erste Panel eröffnete Claudio Radaelli. Er präsentierte seine meta-analytische Perspektive auf die Europäisierungsforschung und gab einen Überblick über die in diesem Kontext zu verortenden, verschiedenen kausalen Ansätze. Daran anschließend beschäftigten sich Thomas König und Hans-Jürgen Bieling mit der Messbarkeit von Europäisierung auf der Polity-Ebene. Während Bieling sich eher allgemein mit der Thematik der Transformation des klassischen Nationalstaates im Zuge der Europäisierung und den Grenzen der Messbarkeit dieser EU-induzierten Transformationsprozesse mithilfe von quantitativen Ansätzen befasste, präsentierte König die Ergebnisse einer Untersuchung darüber, inwiefern die europäische Integration die Beziehungen zwischen dem jeweiligen Parlament und der Regierung zum Vor- oder Nachteil des jeweils anderen beeinflusse. König kam dabei zu dem Ergebnis, dass es kaum Evidenz für die häufig geäuBerte Annahme gebe, dass der Bundestag gegenüber der Bundesregierung im Rahmen der europäischen Einigung geschwächt werde.

Im zweiten Panel wurde anhand von fünf europäischen Mitgliedstaaten der tatsächliche Einfluss der europäischen Gesetzgebung auf die jeweilige nationale Gesetzgebung gemessen. Dabei setzten die Vortragenden unter Berücksichtigung verschiedener länderbedingter $\mathrm{Da}$ tensätze das jeweils auf der europäischen und nationalen Ebene produzierte geltende Recht in Proportion zueinander. Zunächst untersuchte Jørgen Grønnegård Christensen, wie viel der im Jahre 2003 in Dänemark geltenden Gesetzgebung sich prozentual auf die Umsetzung europäischer Rechtsakte zurückverfolgen lässt. Seine Untersuchung beschränkte er dabei auf
Strengths and Weaknesses in Cross-Sectoral Quantitative Approaches

Denmark

Prof. Dr. Jørgen Grønnegård Christensen, Aarhus University

Finland

Prof. Dr. Matti Wiberg, University of Turku

Austria

Marcelo Jenny, MZES, Mannheim

France

Eric Kerrouche, University of Bordeaux

Germany

Prof. Dr. Annette Elisabeth Töller, University of Erfurt

\section{Comment/Discussion}

Prof. Dr. Thomas König, MZES, Mannheim

What Can Cross-Sectoral Analysis Learn From Sectoral Quantitative Approaches?

Culture and Environment

Dr. Wybe Douma, T.M.C. Asser Institute

Environmental and Social Policy

Prof. Dr. Gerda Falkner, Institute for European Integration Research, Austrian Academy of Sciences; University of Vienna

Tax Policy

Dr. Susanne Uhl, DGB, Confederation of German Trade Unions

Transport Policy

Dr. Dieter Plehwe, WZB, Berlin

Comment/Discussion

Prof. Dr. Susanne K. Schmidt, University of Bremen

When Law Meets Politics: Facing the Methodological Challenges

Introduction: Facing the Methodological Challenges

Prof. Dr. Annette Elisabeth Töller, University of Erfurt

Focus on National Law and Law-Making Can We Compare at all? National Styles in Law-Making: A Challenge to Comparative Measurement?

Marcelo Jenny, MZES, Mannheim

Measuring Hierarchy, Complementarities, or Degrees of National Autonomy?

Dr. Oliver Treib, Institute for Advanced Studies, Vienna 
die Umsetzung von EU-Richtlinien und identifizierte europäisierte Gesetze anhand ihrer Referenz zu CELEX-Nummern. ${ }^{2}$ Christensen stellte fest, dass Europäisierung vor allem in solchen Politikfeldern zu beobachten sei, die thematisch mit dem europäischen Binnenmarkt zusammenhingen. Der Gesamteinfluss der Europäisierung der dänischen Gesetzgebung liege entgegen der Delorschen These nur bei 14 Prozent. Ähnliche Ergebnisse präsentierte Matti Wiberg für das Beispiel Finnland. Er überprüfte für den Zeitraum von 1995-2007, wie viel Prozent der finnischen Gesetzgebung eine Referenz zu EU-Normen aufwies und kam dabei auf einen Prozentsatz von 11,4. In Österreich ließen sich nach Angaben von Marcelo Jenny im Jahre 200325 Prozent der österreichischen Gesetzgebung im Gegensatz zu 45 Prozent in der Phase vor dem EU-Beitritt Österreichs im Jahre 1995 auf europäische Richtlinien zurückführen. Dies ging aus einer gemeinsam von Jenny und Wolfgang C. Müller verfassten und im Rahmen des Panels von Jenny präsentierten Studie hervor. Anhand von CELEX-Nummern, Referenzen zu EU-Normen in den Einleitungs- beziehungsweise Schlusssätzen der österreichischen Bundesgesetzgebung, erläuternden Schlussbemerkungen von Gesetzesentwürfen der österreichischen Regierung und von parlamentarischen Debatten hatten Jenny und Müller den Prozentsatz der EU-beeinflussten österreichischen Gesetzgebungsakte ermittelt und dabei versucht, auch die Spezifika des österreichischen politischen Systems und seiner Rechtskultur in die Analyse einzubeziehen. Mit der etwas provokativ formulierten Frage, ob französische Gesetze in Brüssel geschrieben würden, leitete Eric Kerrouche daran anschließend in seine gemeinsam mit Sylvain Brouard und Olivier Costa verfasste - Untersuchung der Europäisierung der französischen Gesetzgebung im
How Far are National Policies Reflected in Laws? On the Limits of Analyzing Legal Acts Prof. Dr. Gunnar Folke Schuppert, WZB, Berlin

Measuring What? Subjects and Sources of Europeanization

What Can We Measure? How Much Complexity Can We Manage?

Dr. Dieter Plehwe, WZB, Berlin

How Far Does Separating Different Types of Law Get Us in Terms of Europeanization Effects?

Prof. Dr. Linda Senden, Tilburg University

How to Interpret the Data We Generate?

Is Less Europeanization More National Autonomy? - And Other Nasty Questions

Prof. Dr. Jørgen Grønnegård Christensen, Aarhus University

Zeitraum von 1986-2003 ein. Wie seine Vorredner konnte auch er nur einen geringen Prozentsatz der in diesem Zeitraum entstandenen französischen Normen auf europäische Richtlinien, internationale Verträge und Vereinbarungen sowie Urteile des Europäischen Gerichtshofes $(\mathrm{EuGH})$ zurückführen. Demnach sei der Prozentsatz der europäisierten französischen Normen seit 1986 von weniger als 3 Prozent auf 10 Prozent im Jahr 1990 und schließlich 13,3 Prozent im Jahr 2003 gestiegen. Nur die Hälfte des europäisierten Rechts betreffe dabei jedoch innenpolitische Belange. Ein Großteil des europäisierten Rechts sei auf die Umsetzung internationaler Vereinbarungen und Verträge zurückzuführen. Annette Elisabeth Töller präsentierte anschließend ihre Ergebnisse für die Bundesrepublik Deutschland. Sie hatte untersucht, welcher Prozentsatz der in den Jahren 1983-2005 entstandenen deutschen Gesetzesakte im Bereich der Innenpolitik einen sogenannten ,europäischen Impuls ${ }^{` 3}$ aufwies. Nach

2 Alle europäischen Rechtsakte werden im EUR-Lex-System der Europäischen Union unter einer bestimmten Kennzahl, der CELEX-Nummer, eingeordnet.

3 Der europäische Impuls ist eine Kategorie im „Dokumentations- und Informationssystem für Parlamentarische Vorgänge“ (DIP) früher Datenbank über den „Stand der Gesetzgebung des Bundes“ (GESTA). Er weist Gesetze aus, die z.B. eine EU-Richtlinie umsetzen oder von Beschlüssen des Ministerrats beeinflusst sind. Vgl. Annette Elisabeth Töller: Mythen und Methoden. Zur Messung der Europäisierung der Gesetzgebung des Deutschen Bundestages jenseits des 80-Prozent-Mythos, in: Zeitschrift für Parlamentsfragen 1/2008, S. 3-17. 
Töller ist der Prozentsatz der europäisierten Gesetzgebung in der Bundesrepublik Deutschland seit 1983 konstant angestiegen. Waren in der 10. Legislaturperiode noch 16,8 Prozent der deutschen Gesetzgebung als europäisiert zu betrachten, so waren es in der 15. Legislaturperiode schon 39,1 Prozent. Zu prüfen sei jedoch, ob sich dieser Trend auch in der laufenden Legislaturperiode fortsetze.

In der an die Länderstudien anknüpfenden Diskussion wurden vor allem die Unterschiede zwischen den einzelnen Analysen deutlich. Zwar waren alle vortragenden Wissenschaftler in ihren Studien zu dem Ergebnis gekommen, dass die Delorsche These mehr Mythos sei als dass sie der Wirklichkeit entspreche und dass der Grad der Europäisierung je nach Politikfeld unterschiedlich ausfalle, doch - darauf verwies König als Moderator des Panels müssten, um die einzelnen Studien vergleichbar zu machen, gleiche Begrifflichkeiten und Methoden verwendet werden. In der Tat hatten sich alle Vortragenden - bedingt auch durch die Spezifika der einzelnen Länder hinsichtlich ihrer politischen Systeme und Kulturen anderer Datensätze, Analyseeinheiten und Zeiträume bedient. Ein weiterer Kritikpunkt, der gleichermaßen alle Studien betraf, wurde von Linda Senden geäußert. Sie bemängelte, dass sich nahezu alle vorgestellten Studien bei der Auswahl der europäischen Datensätze auf europäische Richtlinien konzentrierten und dabei die unmittelbar anwendbaren europäischen Verordnungen zur Gänze ausblendeten. Damit werde - so Senden - einer der Hauptbereiche, innerhalb derer Europäisierung stattfinde, von der Messung ausgeschlossen. Dies könne zu einer Unterschätzung der gemessenen $\mathrm{Eu}$ ropäisierung führen.

Im dritten Panel ging es um die Messung und Messbarkeit von Europäisierung in einzelnen Politikbereichen. Exemplarisch wurden von Wybe Douma die Bereiche Bildung und Umwelt, von Gerda Falkner die Bereiche der Umwelt- und Sozialpolitik, von Susanne Uhl der Bereich der Steuerpolitik und von Dieter Plehwe der Bereich der Verkehrspolitik untersucht.
Douma zeigte am Beispiel der Niederlande auf, dass die Untersuchung und der Vergleich der Europäisierung von bestimmten Politikbereichen möglich sei, und bestätigte die im vorangegangenen Panel geäußerte Annahme, dass der gemessene Prozentsatz dabei zum Teil stark variiere. An dieser Stelle ist vor allem auf die von Uhl im Rahmen des Panels vorgestellte Untersuchung des Politikfelds der Steuern zu verweisen. Uhl kam zu dem erstaunlichen Ergebnis, dass es in diesem Bereich - einem der Herzstücke der mitgliedstaatlichen Souveränität - über die Jahre hinweg zu einer starken Europäisierung gekommen sei. Zwar seien die EU-Mitgliedstaaten in diesem Bereich nach wie vor rechtlich souverän. Doch seien vor allem bei der Erhebung von Mehrwertsteuern und indirekter Steuern sowie bei der Schaffung neuer Steuern und Steuerarten starke Restriktionen von Seiten der Europäischen Union zu beobachten.

In ihren Vorträgen zu den Politikfeldern der Umwelt- und Sozialpolitik sowie der Verkehrspolitik widmeten sich Falkner und Plehwe anschließend vor allem methodischen Fragen. Während Falkner im Rahmen ihrer Studie zu mehr qualitativer Analyse aufrief, versuchte Plehwe dagegen am Beispiel der Verkehrspolitik aufzuzeigen, inwiefern die quantitative Analyse europäischen Rechts geeignet sei, die Entwicklung von Hypothesen über die Transformation der europäischen Governance zuzulassen. In der den Vorträgen folgenden Diskussion wurde vor allem vor der Schwierigkeit der Gleichsetzung von Politikbereichen und politischen Ressorts auf der nationalen Ebene gewarnt. Dies berge das Risiko, dass die jeweiligen Ressorts in ihrer Abgrenzung nicht der tatsächlichen Realität der jeweiligen Politikfelder entsprächen und damit die Messergebnisse verzerren könnten. Außerdem werde der Vergleich mit anderen Zeiträumen oder Legislaturperioden und mit anderen EU-Mitgliedstaaten erschwert.

Im vierten Panel beschäftigten sich die Vortragenden schließlich mit der Frage danach, 
woran Europäisierung überhaupt zu messen und welche Spezifika hier zu beachten seien. Töller verwies in ihren einleitenden Bemerkungen darauf, dass man bei der Messung von Europäisierung auf den nationalen Gesetzgebungsprozess und sein Ergebnis, das geltende Recht, angewiesen sei. Doch stelle sich die Frage, ob das Recht als solches überhaupt als allgemeine und zentrale Analysekategorie verwendbar sei, und inwiefern es hierbei zu länderspezifischen Abweichungen kommen könne. Jenny verwies in seinem Vortrag zunächst darauf, dass es in der Europäischen Union verschiedene Systeme der Gesetzgebung und Arten der Umsetzung europäischer Rechtsakte in nationales Recht, verschiedene rechtliche Kulturen und Verschiedenheiten hinsichtlich des Umfangs der Gesetzgebung gebe, die bei der Messung von Europäisierung berücksichtigt werden müssten. Im Anschluss daran verwies Oliver Treib darauf, dass außer der regulären Umsetzung europäischer Rechtsakte in nationales Recht auch die indirekte Umsetzung europäischen Rechts im Rahmen anderer innenpolitischer Reformen miteinbezogen werden müsste. Gunnar Folke Schuppert nannte anschließend vier weitere Aspekte der Europäisierung nationalen Rechts, die kaum nachweisbar seien und aus diesem Grund bei der Messung häufig unbeobachtet blieben. Schuppert nannte die sogenannte unsichtbare Europäisierung, die zum Beispiel im Rahmen der europarechtskonformen Auslegung nationaler Rechtsakte durch nationale Gerichte deutlich werde. Er verwies auf die kontextabhängige Europäisierung, die beispielsweise in punktuellen Angleichungselementen der deutschen Rechtskultur an das angelsächsische Rechtsdenken bestehe, und nannte drittens die Tatsache, dass nationale Gerichte zum Teil bei und vor ihrer Rechtsprechung die Urteile und Meinungen anderer nationaler Gerichte und des EuGH miteinbezögen. Viertens verwies Schuppert auf die sogenannte informelle Eu- ropäisierung, die zum Beispiel im Rahmen des Vergleichs der eigenen nationalen Reformprozesse mit denen in anderen Mitgliedstaaten auftrete und zu einer Anpassung des eigenen politischen Handelns an letztere führen könnte. Er schloss seinen Vortrag mit der Aussage, dass man aufgrund der großen Reichweite der Europäisierung ihren Grad kaum überschätzen könne. Plehwe kam bei seiner anschließenden Darstellung der verschiedenen Facetten des Rechts zu einer ähnlichen Einschätzung. Um den tatsächlichen Grad von Europäisierung abbilden zu können, müsse man beispielsweise zwischen verschiedenen Arten des Rechts und ihren Inhalten unterscheiden. Ähnlich, aber ungleich kritischer rief im Anschluss daran Senden insbesondere die Politikwissenschaftler nachdrücklich dazu auf, nicht nur quantitativ zu messen, sondern sich auch auf die Natur und den Inhalt von Recht zu fokussieren und beispielsweise eine Unterscheidung von Primär-, Sekundär- und Tertiärrecht vorzunehmen. Bezöge man nur den Umfang der Gesetzgebung in die Analyse ein, so verpasse man einen Großteil des tatsächlichen Europäisierungsprozesses.

Kommen wir zu der eingangs von Radaelli geäußerten These zurück, dass das Forschungsfeld der Europäisierung immer komplexer werde. Diese These ist auch nach dieser sehr vielfältigen und aufschlussreichen Konferenz zu bestätigen. Viele Probleme und Herausforderungen an die Europäisierungsforschung sind während der Konferenz thematisiert worden. $\mathrm{Ob}$ und wie Europäisierung tatsächlich zu messen ist, um zu einer realistischen Einschätzung des 80\%-Mythos zu kommen, kann nicht abschließend beantwortet werden. Festzuhalten ist, dass es für die Forschung - ob quantitativ, qualitativ oder, wie Christensen abschließend forderte, in der Verbindung quantitativer und qualitativer Ansätze - viel zu tun gibt. 